\title{
Blood N-terminal Pro-brain Natriuretic Peptide and Interleukin-17 for Distinguishing Incomplete Kawasaki Disease from Infectious Diseases
}

\author{
Ling Wu, Yuanling Chen, Shiling Zhong, Yunyan Li, Xiahua Dai and Yazhen Di \\ From Department of Pediatric Rheumatoid Immunology, Ningbo Women and Children's Hospital, Zhejiang, China. \\ Correspondence to Dr Yazhen Di, Department of Pediatric Rheumatoid Immunity Ningbo Women and Children's Hospital, \\ 339 Liuting Street, Ningbo, Zhejiang 315012, China.dyz028@163.com \\ Received: September 23, 2014; Initial review: December 15, 2014; Accepted: March 11, 2015.
}

Objective: To explore the diagnostic value of blood N-terminal pro-brain natriuretic peptide (NT-proBNP) and interleukin-17(IL17) for incomplete Kawasaki disease.

Methods: Patients with Kawasaki disease, Incomplete Kawasaki disease and unclear infectious fever were included in this retrospective study. Their clinical features, and laboratory test results of blood NT-proBNP and IL-17 were collected and compared.

Results: 766 patients with complete clinical information were recruited, consisting of 291 cases of Kawasaki disease, 74 cases of incomplete Kawasaki disease, and 401 cases of unclear infectious diseases. When the consistency with indicator 2 and 3 in Kawasaki disease diagnosis criteria was assessed with blood IL-17 $\geq 11.55 \mathrm{pg} / \mathrm{mL}$ and blood NT-proBNP $\geq 225.5 \mathrm{pg} / \mathrm{dL}$ as the

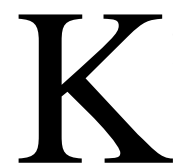

awasaki disease is an acute and immune vascular inflammatory disease with unknown etiology. Incomplete Kawasaki disease has less typical clinical features than the complete type, but it presents much higher incidence of coronary artery lesion [1]. Intravenous immunoglobulin (IVIG) can effectively prevent coronary artery lesions of incomplete Kawasaki disease, but the timing of treatment is very important for its efficacy [2]. Therefore, early diagnosis and treatment is of great significance for incomplete Kawasaki disease. However, the diagnosis of incomplete Kawasaki disease is often delayed due to the lack of typical clinical features and the shortage of specific laboratory diagnostic indicators for the early stage of disease. In the present study, we investigated the clinical features and laboratory indicators in patients with Kawasaki disease, incomplete Kawasaki disease and unknown infectious fever, and studied whether blood Nterminal pro-brain natriuretic peptide (NT-pro BNP) and interleukin-17 (IL-17) could have a diagnostic value for distinguishing incomplete Kawasaki disease and infectious diseases at the early stage.

\section{Methods}

The criteria for the selection of research subjects included: age $\leq 5$ years; diagnosed as Kawasaki disease, criteria, the sensitivity and specificity for distinguishing incomplete Kawasaki disease and infectious diseases reached $86.5 \%$ and $94.8 \%$, respectively. When we chose the consistency with indicator 1 and 2 in Kawasaki disease diagnosis criteria, the appearance of decrustation and/or the BCG erythema, blood IL-17 $\geq 11.55 \mathrm{pg} / \mathrm{mL}$ and blood NT-Pro BNP $\geq 225.5 \mathrm{pg} / \mathrm{dL}$ as the criteria, the sensitivity and specificity for distinguishing incomplete Kawasaki disease and infectious diseases was $43.2 \%$ and $100 \%$, respectively.

Conclusion: Blood NT-proBNP and IL-17 are useful laboratory indicators for distinguishing incomplete Kawasaki disease and infectious diseases at the early stage.

Keywords: Biomarker, Diagnosis, Kawasaki disease, Vasculitis

or incomplete Kawasaki disease, or with fever for $\geq 3$ days with unknown reasons when hospitalized; complete clinical data; confirmed diagnosis when discharged; excluding other non-infectious diseases and implicit diagnosis ultimately; and hospitalized between October 2008 and September 2012. Kawasaki disease was diagnosed as per standard criteria [3]. The infection diagnosis criteria referred to the guidelines formulated by the infectious diseases working party of the German Society of Hematology and Oncology [4]. The research was approved by the hospital's Ethnical Committee, and informed consent obtained from custodians of all participants.

Accompanying Editorial: Pages 473-74.

Data collected included: fever persisting 3-7 days, polymorphous exanthema, conjunctival congestion (no photophobia and no secretions), changes of lips and oral cavity, acute cervical lymphadenopathy (non-purulent, unilateral, diameter $>1.5 \mathrm{~cm}$ ), changes of peripheral extremities: (reddening and indurative edema of palms and soles at the initial stage, and membranous desquamation from fingertips at the recovery phase), decrustation, and BCG erythema. Blood samples of all patients were drawn at the acute stage and on the day of 
admission (3-7 days after the onset, no high dose of intravenous gamma globulin and aspirin therapy). Laboratory tests included NT-proBNP, IL-17, C-reactive protein (CRP), and erythrocyte sedimentation rate (ESR). The level of blood NT-proBNP was quantified using an automated enzyme-linked fluorescence analyzer (Biomerieux Sa Company, miniVIDAS, France) and the matched NT-proBNP reagent. The level of IL-17 was detected by enzyme-linked immunosorbent assay (ELISA) using the IL-17 ELISA Kit (R\&D System, Minneapolis, USA).

SPSS 13.0 software was used for all data analyses. The normally distributed measurement data were analyzed with one-way ANOVA and LSD tests. The count data were analyzed with Fisher's exact test or chi-square test. $P<0.05$ was considered as statistically significant. The value of NT-proBNP was transformed into Napierian logarithm to approach the normal distribution. The resultant average and standard deviation of NT-proBNP were then converted back to their actual values by antilogarithm. Additionally, ROC curve analysis was performed on the single factor of statistical significance and the meaningful clinical features and laboratory indicators were further analyzed to obtain the optimal critical value of incomplete Kawasaki disease identification and diagnosis.

\section{Results}

A total of 766 children (291 with Kawasaki disease and 74 with Incomplete Kawasaki disease) were recruited. 401 children had other infectious diseases. 166 of the Kawasaki disease patients were boys and the mean (SD) age were 2.0 (0.9) (Table I). About 60\% of Kawasaki disease was associated with comorbid infections in this study. The infectious disease patients included 208 cases of respiratory infection (in particular, 109 cases were with lower respiratory infection), 21 cases of digestive tract infection, 31 cases of urinary tract infection, 39 cases of the central nerve system infection, 27 cases of infectious mononucleosis syndrome, 15 cases of lymphadenitis, and 60 cases of sepsis.
TABLE I COMPARISON OF BASELINE AND LABORATORY Parameters Among ChILdREN WITH KaWASAKI DisEASE, INCOMPLETE KAWASAKI DISEASE AND OTHER INFECTIONS

\begin{tabular}{llll}
\hline Parameter & $\begin{array}{l}\text { Kawasaki } \\
\text { disease } \\
(n=291)\end{array}$ & $\begin{array}{l}\text { Incomplete } \\
\text { Kawasaki } \\
\text { disease } \\
(n=74)\end{array}$ & $\begin{array}{l}\text { Infectious } \\
\text { diseases } \\
(n=401)\end{array}$ \\
\hline Age (y) & $2.0(0.9)$ & $1.9(0.9)$ & $2.2(1.1)$ \\
Gender (male), $n(\%)$ & $166(57.0)$ & $41(55.4)$ & $223(55.6)$ \\
NT-proBNP(pg/dL) & $802.5(4.5)$ & $900.4(3.8)$ & $121.0(2.5)^{* \#}$ \\
IL-17 (pg/mL) & $35.4(13.5)$ & $33.8(15.5)$ & $7.7(7.2)^{* \#}$ \\
CRP (mg/L) & $68.7(51.4)$ & $63.5(41.9)$ & $35.9(33.2)^{* \#}$ \\
ESR (mm/h) & $59.8(31.1)$ & $61.4(31.4)$ & $27.8(14.2)^{* \#}$ \\
\hline
\end{tabular}

*Compared with Kawasaki disease group $P<0.05$; ${ }^{*}$ Compared with Incomplete Kawasaki disease group $P<0.05$; All values in mean (SD).

The levels of blood NT-probing, IL-17, CRP and ESR were very similar in Kawasaki disease and incomplete Kawasaki disease groups, but all were significantly lower in infectious group $(P<0.05$, Table $\mathbf{I})$. The areas below the ROC curves of the levels of blood NT-proBNP and IL-17 were respectively 0.906 and 0.960 (Table II and Fig. 1). The diagnostic cut-off of blood NT-proBNP blood IL-17, blood CRP (DCP) and ESR, and the corresponding sensitivity and specificity for identifying incomplete Kawasaki disease and infectious diseases is shown in Fig. 1.

We finally combined the clinical manifestations and laboratory results for distinguishing incomplete Kawasaki disease and infectious diseases. When we chose the consistency with indicator 2 and 3 in Kawasaki disease diagnosis criteria, blood IL-17 $\geq 11.55 \mathrm{pg} / \mathrm{mL}$ and blood NT-proBNP $\geq 225.5 \mathrm{pg} / \mathrm{dL}$ as the criteria, the sensitivity and specificity for distinguishing incomplete Kawasaki disease and infectious diseases reached $86.5 \%$ (64/74) and 94.8\% (380/401), respectively. When we chose the consistency with indicator 1 and 2 in Kawasaki disease diagnosis criteria, the appearance of decrustation and (or) the BCG Erythema, blood IL-17 $\geq 11.55$ pg/mL

TABLE II Diagnostic Values of Blood NT-PRoBNP, IL-17, CRP And ESR in Distinguishing Incomplete KaWASAKi Disease AND INFECTIOUS DISEASES

\begin{tabular}{llcccr}
\hline Parameter & $\begin{array}{l}\text { Diagnostic } \\
\text { cut-off }\end{array}$ & $\begin{array}{c}\text { Sensitivity } \\
(\%)\end{array}$ & $\begin{array}{c}\text { Specificity } \\
(\%)\end{array}$ & $\begin{array}{c}\text { Area-under } \\
\text { the curve }\end{array}$ & $\begin{array}{c}\text { P } \\
\text { NT-proBNP(pg/dL) }\end{array}$ \\
$\geq 225.5$ & 89.2 & 84.0 & 0.906 & $<0.001$ \\
IL-17 (pg/mL) & $\geq 11.55$ & 91.9 & 88.8 & 0.960 & $<0.001$ \\
CRP $(\mathrm{mg} / \mathrm{L})$ & $\geq 38.05$ & 74.3 & 72.1 & 0.731 & $<0.001$ \\
ESR $(\mathrm{mm} / \mathrm{h})$ & $\geq 44.5$ & 70.3 & 88.3 & 0.840 & $<0.001$ \\
\hline
\end{tabular}




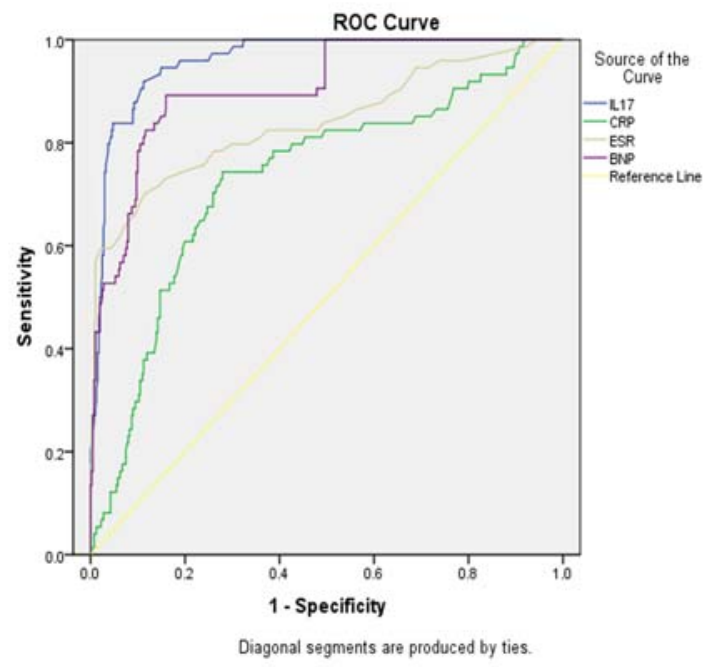

FIG. 1 ROC curves of blood NT-proBNP, IL-17, CRP and ESR for distinguishing Incomplete Kawasaki disease and infectious diseases.

and blood NT-proBNP $\geq 225.5 \mathrm{pg} / \mathrm{dL}$ as the criteria, the sensitivity and specificity for distinguishing incomplete Kawasaki disease and infectious diseases reaches 43.2\% (32/74) and 100\% (401/401), respectively.

\section{Discussion}

In this retrospective record-review, we found that the blood NT-proBNP levels in Kawasaki disease and incomplete Kawasaki disease are higher than that in infectious diseases. Recent studies also report that the level of blood NT-proBNP is of great significance in diagnosing incomplete Kawasaki disease at an early time $[5,6]$. Secreted NT-proBNP is increased in Kawasaki disease and incomplete Kawasaki disease because of the partial inflammatory damages of cardiac muscle, and also increased synthesis in the ventricular myocytes stimulated by some inflammatory factors $[7,8]$.

We found a much higher level of blood IL-17 in Kawasaki disease and incomplete Kawasaki disease compared with infectious disease. IL-17, as an inflammatory factor, guides neutrophil and monocyte towards vascular wall to catalyze the reaction of inflammations and participate in the emergence of multiple self-immune diseases and inflammatory responsive diseases, for example systemic lupus erythematosus [9], systemic onset juvenile idiopathic arthritis [10] and Kawasaki disease [11], by inducing the penetration and damages of blood vessel tissues [12-14]. It seems that the combination of blood IL-17 and NTproBNP further improves the specificity for the diagnosis of incomplete Kawasaki disease.
In children with fever of more than five days without a diagnosis, accompanied by indicator 1 and 2 of Kawasaki disease diagnosis criteria (decrustation and/or BCG erythema), and blood IL-17 $\geq 11.55 \mathrm{pg} / \mathrm{mL}$ and blood NTprobing $\geq 225.5 \mathrm{pg} / \mathrm{dL}$; the specificity for distinguishing incomplete Kawasaki disease and infectious diseases reached almost $100 \%$. Therefore, even if the patients are at the early stage and have no coronary artery lesions, based on decrustation and or BCG erythema, and abnormally high blood IL-17 and NT-proBNP levels, they can be reasonably diagnosed with incomplete Kawasaki disease.

Contributors: All authors have contributed, designed and approved the study.

Funding: Zhejiang Medical and Hygienic Scientific Research Fund (2008B168); Competing interests: None stated.

\section{REFERENCES}

1. Sudo D, Monobe Y, Yashiro M, Mieno MN, Uehara R, Tsuchiya K, et al. Coronary artery lesions of incomplete Kawasaki disease: a nationwide survey in Japan. Eur J Pediatr. 2012;171:651-6.

2. Muta H, Ishii M, Yashiro M, Uehara R, Nakamura Y. Late intravenous immunoglobulin treatment in patients with Kawasaki disease. Pediatrics. 2012;129:e291-7.

3. Newburger JW, Takahashi M, Gerber MA, Gewitz MH, Tani LY, Burns JC, et al. Diagnosis, treatment, and longterm management of Kawasaki disease: a statement for health professionals from the Committee on Rheumatic Fever, Endocarditis, and Kawasaki Disease, Council on Cardiovascular Disease in the Young, American Heart Association. Pediatrics. 2004;114:1708-33.

4. Penack O, Beinert T, Buchheidt D, Einsele H, Hebart H, Kiehl MG, et al. Management of sepsis in neutropenia: guidelines of the infectious diseases working party (AGIHO) of the German Society of Hematology and Oncology (DGHO). Ann Hematol. 2006;85:424-33.

5. Cho SY, Kim Y, Cha SH, Suh JT, Han MY, Lee HJ. Adjuvant Laboratory Marker of Kawasaki Disease; NTpro-BNP or hs-CRP. Ann Clin Lab Sci. 2011;41:360-3.

6. No SJ, Kim DO, Choi KM, Eun LY. Do predictors of incomplete Kawasaki disease exist for infants? Pediatr Cardiol. 2013;34:286-90.

7. Iwashima S, Ishikawa T. B-type natriuretic peptide and Nterminal pro-BNP in the acute phase of Kawasaki disease. World J Pediatr. 2013;9:239-44.

8. Di Yazhen, Wu Ling, Li Yunyan, Ma Tianrui, Wang Tianbo, Lin Yahong, et al. The clinical value of plasma Nterminal pro-brain natriuretic peptide and serum albumin in the early diagnosis of incomplete Kawasaki disease. Chinese J Rheumatol. 2013;9:595-600.

9. Rana A, Minz RW, Aggarwal R, Anand S, Pasricha N, Singh S. Gene expression of cytokines (TNF-alpha, IFN-gamma), serum profiles of IL-17 and IL-23 in paediatric systemic lupus erythematosus. Lupus. 2012;21:1105-12.

10. Omoyinmi E, Hamaoui R, Pesenacker A, Nistala K, Moncrieffe $\mathrm{H}$, Ursu S, et al. Th1 and Th17 cell 
subpopulations are enriched in the peripheral blood of patients with systemic juvenile idiopathic arthritis. Rheumatology (Oxford). 2012;51:1881-6.

11. Jia S, Li C, Wang G, Yang J, Zu Y. The T helper type 17/ regulatory $\mathrm{T}$ cell imbalance in patients with acute Kawasaki disease. Clin Exp Immunol. 2010;162:131-7.

12. Jadidi-Niaragh F, Mirshafiey A. The deviated balance between regulatory $\mathrm{T}$ cell and Th17 in autoimmunity.
Immunopharmacol Immunotoxicol. 2012;34:727-39.

13. Maddur MS, Miossec P, Kaveri SV, Bayry J. Th17 cells: biology, pathogenesis of autoimmune and inflammatory diseases, and therapeutic strategies. Am J Pathol. 2012;181:8-18.

14. Miossec P, Kolls JK. Targeting IL-17 and TH17 cells in chronic inflammation. Nat Rev Drug Discov. 2012;11:763-76. 